\title{
Shape-Driven Segmentation of the Arterial Wall in Intravascular Ultrasound Images
}

\author{
Gozde Unal, Senior Member, IEEE, Susann Bucher, Stephane Carlier, Greg Slabaugh, Tong Fang, and Kaoru Tanaka
}

\begin{abstract}
Segmentation of arterial wall boundaries from intravascular images is an important problem for many applications in the study of plaque characteristics, mechanical properties of the arterial wall, its 3-D reconstruction, and its measurements such as lumen size, lumen radius, and wall radius. We present a shape-driven approach to segmentation of the arterial wall from intravascular ultrasound images in the rectangular domain. In a properly built shape space using training data, we constrain the lumen and media-adventitia contours to a smooth, closed geometry, which increases the segmentation quality without any tradeoff with a regularizer term. In addition to a shape prior, we utilize an intensity prior through a nonparametric probability-densitybased image energy, with global image measurements rather than pointwise measurements used in previous methods. Furthermore, a detection step is included to address the challenges introduced to the segmentation process by side branches and calcifications. All these features greatly enhance our segmentation method. The tests of our algorithm on a large dataset demonstrate the effectiveness of our approach.
\end{abstract}

Index Terms-Arterial wall segmentation, calcification detection, intensity prior, intravascular ultrasound (IVUS), lumen segmentation, media adventitia segmentation, model-based segmentation, side branch detection, shape prior.

\section{INTRODUCTION}

I NTRAVASCULAR ULTRASOUND (IVUS) allows realtime tomographic assessment of the arterial wall, which is very important to study vascular wall architecture for diagnosis and assessment of the progression of the cardiovascular diseases [1]. By the American Heart Association Heart Disease and Stroke Statistics, cardiovascular disease is the leading cause of death in the United States, and the coronary artery disease has the highest percentage (53\%) of death among the heart diseases [2].

Atherosclerosis is a disease characterized by a deposit of plaque in an arterial wall over time. The disruption of an atherosclerotic plaque is considered to be the most frequent

Manuscript received February 19, 2007; revised October 25, 2007.

G. Unal is with the Faculty of Engineering and Natural Sciences, Sabanci University, Istanbul 34956, Turkey (e-mail: gozdeunal@ sabanciuniv.edu).

S. Bucher was with Siemens Corporate Research, Princeton, NJ 08540 USA. She is now with Avaloq Evolution, AG, Zürich, Switzerland (e-mail: susann. bucher@gmail.com).

G. Slabaugh and T. Fang are with Siemens Corporate Research, Princeton, NJ 08540 USA (e-mail: greg.slabaugh@gmail.com; tong.fang@siemens.com).

S. Carlier was with the Division of Cardiology, Department of Medicine, Cloumbia University, New York, NY 10032 USA. He is now with the Intravascular Imaging and Physiology, Cardiovascular Research Foundation, New York, NY 10022, USA (e-mail: scarlier@crdbe.jnj.com).

$\mathrm{K}$. Tanaka is with the Intravascular Imaging and Physiology, Cardiovascular Research Foundation, New York, NY 10022 USA.

Color versions of one or more of the figures in this paper are available online at http://ieeexplore.ieee.org.

Digital Object Identifier 10.1109/TITB.2008.920620
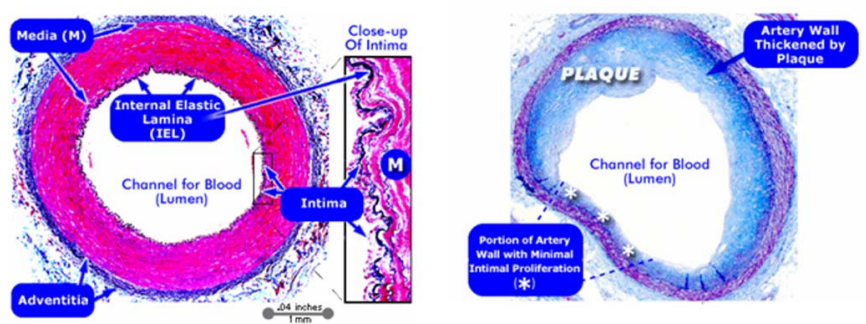

Fig. 1. Cross-sectional microscopic views. Left: normal coronary artery; right: coronary artery with atherosclerotic plaque [Copyright (c) 1996-2005, WebMD, Inc. All rights reserved].

cause of heart attack and sudden cardiac death. Studying vulnerable plaques constitutes a major research area in the field of clinical and medical imaging. In order to track progression and regression during therapy of the atherosclerosis, the inner and outer borders of the arterial wall are extracted and the plaque area is identified in the region between these two borders (see Fig. 1 for both healthy and diseased arterial wall cross-sectional views).

A catheter is inserted through the patient's groin into an artery and pushed toward the distal end of the coronary arteries. Thereafter, the ultrasound transducer in the catheter is pulled back with constant speed. During the pullback, a sequence of IVUS images are acquired. In Fig. 1, the lumen is the interior of the vessel, through which the blood flows. The intima is the innermost layer of an artery. It is made up of one layer of endothelial cells and is supported by an internal elastic intima. The endothelial cells are in direct contact with the blood flow. It is a fine, transparent, colorless structure that is highly elastic. The media is the middle layer of an artery, which is made up of smooth muscle cells and elastic tissue. The adventitia is the outermost layer of the blood vessel, surrounding the media. It is mainly composed of collagen. Extraction of the boundaries of the coronary arterial wall by segmenting the lumen and mediaadventitia contours is a first step in measuring quantities such as lumen diameter and plaque dimensions, and assessment of the atherosclerotic plaque. Manual segmentation and processing of the lumen contour and the media-adventitia contour is tedious, time-consuming, and susceptible to intra- and interobserver variability. Due to the high number of images in a typical IVUS pullback, typically in the order of hundreds, automated segmentation of the arterial contours is an essential task.

Numerous approaches have been used to (semi-) automatically detect regions of interest in IVUS images. Most reported successful approaches are based on contour detection using a minimization of a cost function of the boundary contours 
or deformable models. Various optimization algorithms are applied. One approach was graph searching [3] in which prior knowledge of the expected IVUS pattern is incorporated. In another earlier IVUS segmentation method, simulated annealing was utilized [4], and a dynamic programming approach was used in [5]. Active contour and surface models particularly played an important role in IVUS segmentation, since salient features in IVUS such as lumen boundary and media-adventitia border can be described conveniently by closed contours. In [6], a contour is propagated along the radial direction with a balloon force. Others utilized active contours through a neighborhood search [7], [8], active contours with a statistical distribution of blood and vessel wall tissue [9], [10], and a knowledge-based approach that incorporates shadow, calcified plaque, and side branch information in a high-level system [11].

The most widely used and simplest image descriptor that was used is the image gradient. However, there are various challenges for automated IVUS segmentation techniques that are caused by image noise in IVUS such as speckle, shadowing due to calcium deposits, or implanted stent, and image artifacts such as mis-registered echoes, or signal loss, and presence of a guide wire. To overcome noise effects, various image descriptors such as smoothed image gradients, local intensity information, edge contrast [12], textural properties [13], statistical properties of speckle through a Rayleigh distribution [12], [14] have been utilized. However, assumption of a priori knowledge about the speckle pattern in IVUS through a parametric distribution such as Rayleigh or mixture of Gaussians as in [9], [10], [14], and [12] might not be applicable in all cases.

Active contour methods based on a combination of transversal and longitudinal contour detection techniques [6], [7] exploit the similarity of sequential frames. These methods attempted to incorporate the relation and connectivity of the frames in the nontransversal dimension in order to segment the entire sequence of the images at the same time. However, usually initial contours must be placed on the first slice, last slice, and many intermediate slices for the algorithms to run three dimensionally. Also, even though these algorithms are called 3-D, most of them affect the image-based forces in only the transverse plane for vessel wall segmentation and only utilize 3-D smoothness and continuity constraints through a 2-D contour in an adjacent frame. In [15], a probabilistic lumen segmentation using elliptic templates was proposed. This is the first shape-based approach to segmenting the lumen contour in IVUS images, and using such a restricted deformable shape made their model generally more stable toward image artifacts. However, in many situations, the lumen borders may not be represented by such a simple shape as depicted in Fig. 2. Similarly, it can be observed that the vessel border sometimes exhibits subtle local variations that may not be approximated by an ellipse model.

Most of the techniques presented in the literature looked into lumen contour extraction alone. In this paper, we target extraction of both the lumen and the media contours. Some limitations with most of the proposed algorithms can be listed as the need for an initialization of the contour that may require manually placing markers along the searched contour to guide the segmentation, or markers on selected frames along the 3-D pull-

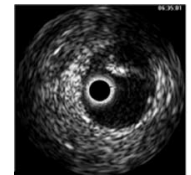

(a)

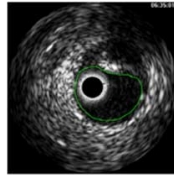

(b)

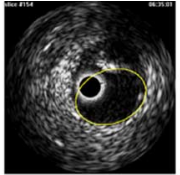

(c)

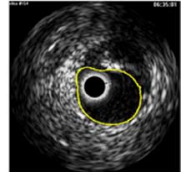

(d)
Fig. 2. (a) IVUS frame. (b) Manual outline of the lumen border. (c) Ellipse cannot approximate the lumen border. (d) Proposed algorithm outlines the lumen reasonably well.

back sequence, or selection of a region of interest, whereas our algorithm targets a fully automatic estimation of the lumen and media contours.

None of the previous IVUS segmentation algorithms used a statistical shape-driven approach, as we present in this paper. Medical IVUS image segmentation algorithms are almost always hampered by noise, stents, shadowing due to calcium deposits, and therefore, have to cope with weak or missing boundaries of structures. Under such conditions, prior models proved to be useful in aiding the segmentation process. In fact, active shape models (ASMs) have become a popular tool in various segmentation applications for prostate, heart structures such as the left ventricle, and brain structures such as the corpus callosum [16]-[19]. The first ASMs [18] used parametric point distribution models learned through principal component analysis (PCA). The contours or shapes in a training dataset are first aligned to build an average shape, and eigen modes or eigenshapes obtained through PCA describe the variations from the mean shape. Implicit shape representations are now more popular since they solve the correspondence problem between shapes during the alignment stage [16], [19].

\section{A. Our Contribution}

Our first contribution in this paper is a shape-driven approach to IVUS segmentation. We will model both the lumen and media-adventitia contour variations within a shape space, in the "resampled" rectangular domain. Hence, we constrain the lumen and media-adventitia contours to a smooth, closed geometry, which increases the segmentation quality without any tradeoff with a regularizer term, yet with adequate flexibility.

Our second contribution is for the lumen segmentation to utilize a nonparametric intensity model based on a probabilitydensity-based image energy. Furthermore, we incorporate global image measurements into this intensity model rather than pointwise measurements used in previous methods. The mediaadventitia is segmented by employing edge information. We define an oriented smooth gradient that overcomes noise present in IVUS images. In addition, we develop a method to detect calcifications and branch openings, taking advantage of anatomical characteristics. Incorporating the feature information into the media-adventitia contour extraction greatly enhances our segmentation method. The tests of our algorithm on a large dataset demonstrate the effectiveness of our approach. ${ }^{1}$

\footnotetext{
${ }^{1}$ An earlier preliminary version of this paper is published in the Proceedings of MICCAI: The 1st International Workshop on Computer Vision for Intravascular and Intracardiac Imaging [20].
} 


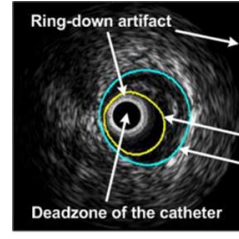

(a)

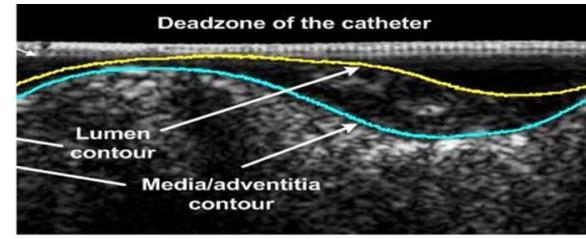

(b)
Fig. 3. Lumen and media-adventitia contours. (a) Display domain. (b) Rectangular domain.

The organization of this paper is as follows. Section II explains our shape representation and building of the shape space. Section III presents our segmentation framework. Section IV describes the feature detection for side branch and calcification features and incorporation of those into the segmentation framework. Finally, Section V presents the results, discussions, and conclusions.

\section{Shape Representation AND Shape Space}

In this paper, we build a statistical shape model to represent the inner and outer arterial wall contours in a compact way, and use this shape prior to drive the segmentation. The details of this method particular to the IVUS application are explained next.

\section{A. 2-D Curves in "Resampled Rectangular" Domain}

To build a statistical shape model, first a shape representation has to be selected. Typical lumen and media-adventitia (ma) contours are shown in Fig. 3 in both the display domain and the resampled rectangular domain, which is the original acquisition format. We choose the rectangular representation, since computations are much simpler due to the 1-D appearance of the segmenting contours [see Fig. 3(b)].

Therefore, in the rectangular image domain $\Omega \in \mathbb{R}^{2}$, we utilize an implicit shape representation by embedding periodic contours $C \in \Omega$ implicitly as the zero-level set of a signed distance function $\Phi: \mathbb{R}^{2} \longrightarrow \Omega$

$$
\boldsymbol{C}=\{(x, y) \in \Omega \mid \boldsymbol{\Phi}(x, y)=0\}
$$

where $\boldsymbol{\Phi}(x, y)<0$ is above (inside) and $\boldsymbol{\Phi}(x, y)>0$ is below (outside) the contour.

\section{B. Data Description}

Twenty in vivo pullbacks of 2176 frames in total were acquired with a Volcano Therapeutics IVG3 machine at 15 frames/s with $0.5 \mathrm{~mm} / \mathrm{s}$ using a $20 \mathrm{MHz}$ transducer. The pullbacks were taken from the left anterior descending artery (LAD), the right coronary artery (RCA), and the left circumflex coronary artery (LCX) (see the heart and the coronary arteries diagrams in Fig. 4).

We used 257 frames from two patient pullbacks to train our shape space and 1919 frames from 18 patient pullbacks for testing. The two pullbacks used for training were not included in the testing phase. The lumen and $\mathrm{m}$-a contours were segmented
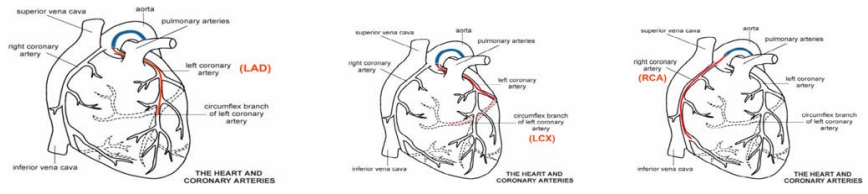

Fig. 4. Heart and the coronary arteries. Highlighted in each figure: LAD, LCX, and RCA.

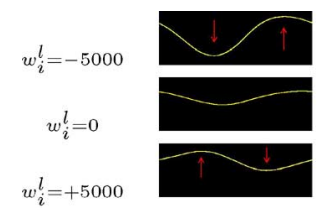

$i=1$

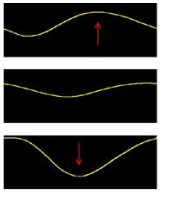

$\mathrm{i}=2$

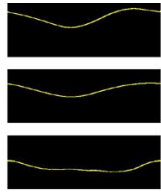

$i=3$

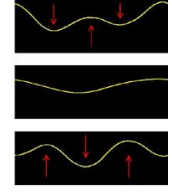

$\mathrm{i}=4$
Fig. 5. Luminal mean shape with weight variations for the first four modes.

by an expert interventional cardiologist in our team, in order to provide the "ground truth" segmentation contours for both training and testing.

\section{Building a Shape Space}

After the signed distance representations for the $N$ lumen shapes $\Phi_{1}^{l}, \ldots, \Phi_{N}^{l}$, and m-a shapes $\Phi_{1}^{a}, \ldots, \Phi_{N}^{a}$ in the training dataset are formed, we conduct statistical analysis. We align all the shapes radially by cropping them from the uppermost row (radial) coordinate where the contours can start, and the lowermost row coordinate where the $\mathrm{m}$-a contours can end. The horizontal (angular) shift of the shape in the rectangular grid corresponds to a rotation of the shape in the display domain. We have not carried out an alignment in the horizontal direction because we would like the shape space to capture the angular shifts through its eigenshapes.

We compute the mean lumen shape: $\Phi_{\text {mean }}^{l}=1 / N \sum_{i=1}^{N}$ $\boldsymbol{\Phi}_{\boldsymbol{i}}^{\boldsymbol{l}}$, and the mean m-a shape: $\boldsymbol{\Phi}_{\text {mean }}^{\boldsymbol{a}}=1 / N \sum_{i=1}^{N} \boldsymbol{\Phi}_{\boldsymbol{i}}^{\boldsymbol{a}}$. We subtract them from each shape in the training set to construct the shape variability matrices $S^{l}=\left[\tilde{\boldsymbol{\Phi}}_{1}^{l} \ldots \tilde{\boldsymbol{\Phi}}_{N}^{l}\right]$ and $\boldsymbol{S}^{a}=\left[\tilde{\boldsymbol{\Phi}}_{1}^{a} \ldots \tilde{\boldsymbol{\Phi}}_{N}^{a}\right]$. After PCA is carried out on both $\boldsymbol{S}^{\boldsymbol{l}}$ and $S^{a}$, we obtain our models that represent each shape as variations around the mean

$$
\boldsymbol{\Phi}^{l}(\boldsymbol{w})=\boldsymbol{\Phi}_{\text {mean }}^{l}+\sum_{i=1}^{k} \boldsymbol{w}_{i}^{l} \boldsymbol{U}_{\boldsymbol{i}}^{\boldsymbol{l}}
$$

where $w^{l}=\left\{w_{1}^{l}, w_{2}^{l}, \ldots, w_{k}^{l}\right\}$ are the weights associated with the first $k$ principal modes $\boldsymbol{U}_{\boldsymbol{i}}^{\boldsymbol{l}}$. The same analysis is carried out for the training set containing the m-a shapes. One can note that shape variations of the vessel in the IVUS images are primarily due to angulation and movement of catheter within the lumen, and anatomical changes such as change of the lumen area and plaque formation.

In Fig. 5, the eigenshapes representing the first four variations in the lumen shape space are shown by varying them around the mean luminal shape: $\boldsymbol{\Phi}_{\text {mean }}^{l}+w_{i}^{l} \boldsymbol{U}_{\boldsymbol{i}}^{\boldsymbol{l}}$, with $i=\{1,2,3,4\}$. Note that the first mode of deformation corresponds to a negative/positive amplification of the curving, the second mode to a radial shift (distance to the catheter) although it also includes 


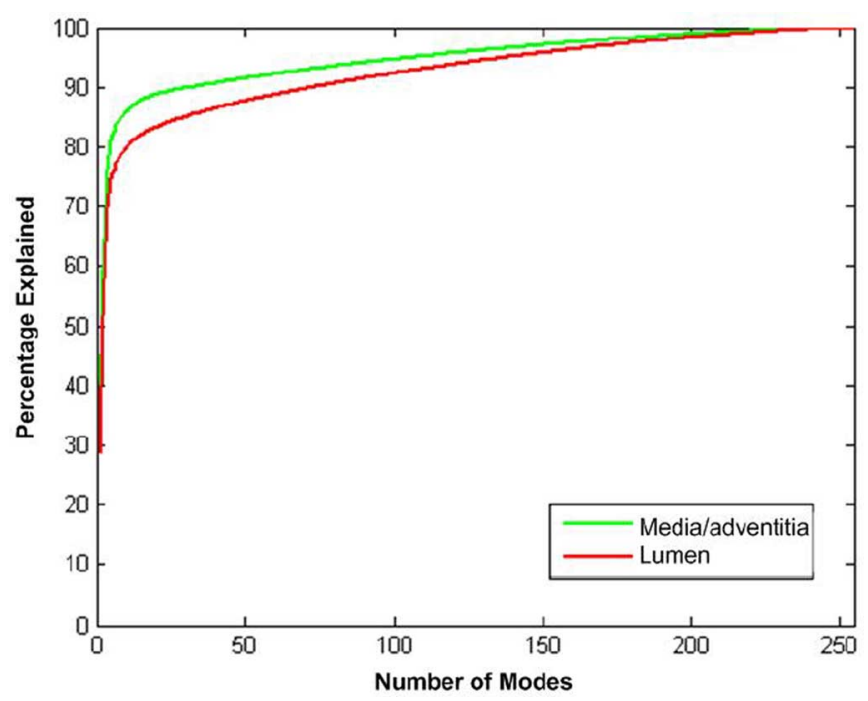

Fig. 6. Modes versus percentage of explained shapes.

an angular shift effect. The third mode corresponds to a flattening versus curving. We note that first three modes include variation for the change of the lumen cross-sectional area as well. The fourth and higher modes explain more local variations of the shape. The same interpretations can be made for the eigenshapes of the m-a contours. As we can see from Fig. 5, the arterial wall contours form a fairly restricted class of shapes, therefore, a small number of eigenshapes $\left\{\boldsymbol{U}_{\boldsymbol{i}}^{\boldsymbol{l}}\right\}_{i=1}^{k}$ and $\left\{\boldsymbol{U}_{\boldsymbol{i}}^{\boldsymbol{a}}\right\}_{i=1}^{k}$ are needed to explain its variations.

Fig. 6 shows the number of modes versus the percentage of variation explained in the shape space. In our experiments, we found that six principal modes suffice for lumen and ma, respectively, to obtain shapes that are both meaningful and smooth. Six modes explain $72.1 \%$ of the luminal shapes and $82.6 \%$ of the m-a shapes.

\section{Preprocessing}

In IVUS images, the catheter creates a dead zone in the center of the display domain, or equivalently, at the top rows of the rectangular domain, along with the imaging artifacts due to "halo" ring-down effects of the catheter (see Fig. 3). Usually, these artifacts should be removed or otherwise they will hamper the segmentation process. The most basic and straightforward approach, that is, to subtract the average image from every frame, does not remove the artifact properly and degrades the image quality in our experimental dataset. For our data, we observe that the artifact stays approximately constant over the IVUS sequence of frames. However, the variance of the artifact is not zero because the luminal border often interferes with the artifact zone. By taking the minimum image $I_{\min }(x, y)=\min _{i \in \lambda} I_{i}(x, y)$ over a set of frames $I_{i}$ of the IVUS sequence $\lambda$ (e.g., 20 frames), we get the constant artifact zone without the bright interferences with the contour [see Fig. 7(b)]. The sums over the rows of $I_{\min }$ are computed and the global maximal row sum is detected. The catheter artifact line is noted down as the first row at which the row sum falls below $50 \%$ of

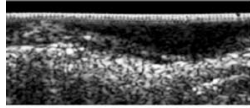

(a)

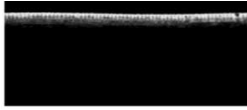

(b)

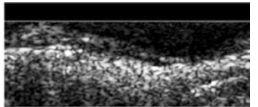

(c)
Fig. 7. (a) Catheter and imaging artifacts in the top rows of the rectangular domain.(b) Minimum image taken over 20 frames. (c) Artifact removed image with the artifact line marked.

the maximal row sum. The rows above this artifact line are omitted from any kind of computations involved in the segmentation. The first local minimum after the global maximum indicates the end of the artifact. The artifact zone of $I_{\min }$ is subtracted from every frame, as shown in Fig. 7(c).

\section{SEGMENTATION FRAMEWORK}

Once we build the statistical shape space, any arterial wall can be represented by a vector of weights $w_{1}^{l}, \ldots, w_{k}^{l}$ associated with the first $k$ principal modes of the lumen data, and $w_{1}^{a}, \ldots, w_{k}^{a}$ of the m-a data. The weights of any shape can be found using (2) by projecting the shape matrix without the mean onto the mode matrix

$$
\boldsymbol{w}^{l}=\boldsymbol{U}_{l}^{T}\left(\boldsymbol{\Phi}^{l}-\boldsymbol{\Phi}_{\text {mean }}^{l}\right)
$$

where $\boldsymbol{U}_{l}$ represents the truncated mode matrix in the reduced subspace. With this compact shape description, we will evolve the shape weights $\boldsymbol{w}$ directly to deform the contour toward the lumen and the m-a borders in the IVUS image. The steps of the segmentation are explained next.

\section{A. Lumen Segmentation}

In this section, we describe the segmentation of the border between the lumen and the inner arterial wall.

1) Initialization of Shape Pose: The first step is to initialize the lumen contour in a way that takes advantage of the prior information about the lumen region having less ultrasound reflection, hence, a darker intensity profile. We shift the mean lumen shape $\boldsymbol{\Phi}_{\text {mean }}^{l}$ in angular direction to minimize the mean intensity above the contour. This simple strategy for the initial shape pose works well, as shown in Fig. 10(c).

2) Evolution of the Shape: For the evolution of the luminal contour, we utilize region statistics from IVUS images for segmentation, and define the following energy

$$
\begin{aligned}
E_{\text {lumen }}\left(\boldsymbol{w}^{l}\right)= & \int_{\Omega}-\mathcal{X}_{\text {in }}(\boldsymbol{x}) \log \left(P_{\text {in }}(I(\boldsymbol{x}))\right) d \boldsymbol{x} \\
& +\int_{\Omega} \mathcal{X}_{\text {out }}(\boldsymbol{x}) \log \left(P_{\text {out }}(I(\boldsymbol{x}))\right) d \boldsymbol{x}
\end{aligned}
$$

where $\mathcal{X}$ is an indicator function for inside and outside the contour. This is the general region-based probabilistic energy functional described in [21]. We model the probability distributions $P_{\text {in }}$ and $P_{\text {out }}$ nonparametrically since we would like to preserve a certain level of generality for our segmentation technique (as the assumption of parametric distributions such as Rayleigh for inside and outside the lumen region may not always hold). 


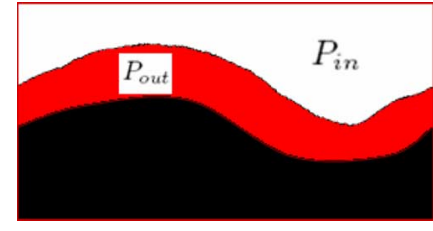

Fig. 8. Regions above and below the lumen contour are used to estimate the intensity probability distributions $P_{\text {in }}$ and $P_{\text {out }}$.

The blood, which flows in the lumen, has low echogenicity; therefore, the lumen region reflects the ultrasound signals less and has speckle of smaller size and lower intensity. The probability distribution inside the lumen, and outside the lumen in a narrowband, e.g., with a radius of 10 (see Fig. 8) can be estimated using intensities in our training dataset. To estimate the nonparametric probability distributions $P_{\text {in }}$ and $P_{\text {out }}$, we use the Parzen windowing technique also called the kernel density estimator: $P(q)=1 / n \sigma \sum_{i=1}^{n} K\left(q-q_{i} / \sigma\right)$, where $n$ is the number of pixels inside or outside the contour and $K(p)=1 / \sqrt{2 \pi} \exp \left(-p^{2} / 2\right)$ is the Gaussian kernel, with a heuristically chosen $\sigma$ value (e.g., $\sigma=10$ ).

Finally, we take the first variation of the energy in (4) to find the gradient flow of the contours represented by the weight vector $\boldsymbol{w}$. The Euler-Lagrange equations result in the ordinary differential equation (ODE):

$$
\frac{\partial w_{i}^{l}}{\partial t}=\int_{\boldsymbol{C}} \frac{-\log \left(P_{\text {in }}(I(\boldsymbol{x}))\right.}{P_{\text {out }}(I(\boldsymbol{x}))} \boldsymbol{U}_{i}^{l} d \boldsymbol{x}
$$

where $\boldsymbol{U}_{i}^{l}$ is the eigenshape corresponding to the $i$ th lumen contour weight $w_{i}^{l}$.

After the initial shape has been found, we repeat the following steps iteratively until the algorithm converges.

1) Initialize the first shape $\boldsymbol{\Phi}_{\text {init }}^{l}$.

2) Compute the weights $w_{i}^{l}(t)$ at time $t=0$ from the shape, using (3).

3) Update the weights using the shape gradients $\partial w_{i}^{l} / \partial t$ in (5)

$$
w_{i}^{l}(t+1)=w_{i}^{l}(t)+\alpha\left[\delta \frac{\partial w_{i}^{l}}{\partial t}(\mathrm{t}-1)+(1-\delta) \frac{\partial w_{i}^{l}}{\partial t}(\mathrm{t})\right]
$$

where the parameter $\alpha$ defines our step size, and the first term in square parenthesis is a momentum term to improve the speed of convergence with $\delta \in[0,1]$.

4) Compute the shape from the weights, using (2).

5) Go to step 3) until algorithm converges.

The segmentation equation (5) uses pointwise intensity measurements on the contour, therefore occasionally may be hampered by speckle noise in the lumen. We design a more global intensity term $I_{\text {modified }}$ that prevents the contour from getting stuck in local minima. This intensity utilizes a maximum function of the averaged intensity over the column above the contour position $\boldsymbol{x}=(x, y)$

$$
I_{\text {modified }}(x, y)=\max _{i \in[0, y]} \frac{1}{y-i+1} \sum_{k=i}^{y} I(x, k)
$$

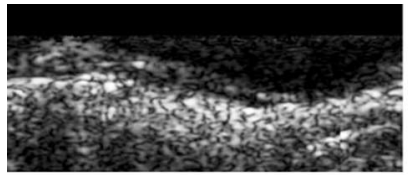

(a)

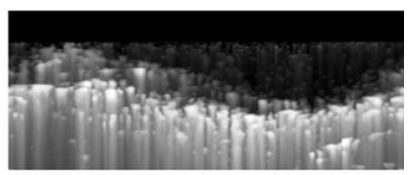

(b)
Fig. 9. (a) Original intensity image. (b) Modified intensity image.

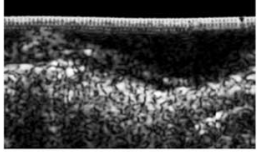

(a)

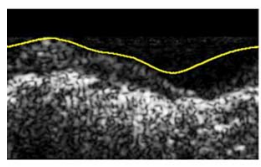

(d)

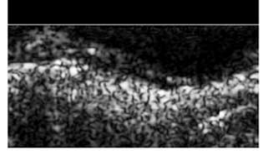

(b)

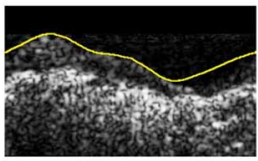

(e)

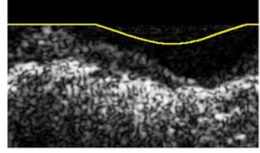

(c)

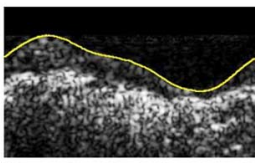

(f)
Fig. 10. (a)and (b) Rectangular domain before and after artifact removal. (c) Initialization of the luminal contour. (d)-(f) Evolution of the luminal contour after three, six, and nine iterations.

where the origin of the image $(0,0)$ is in the top left corner. We then replace $I(\boldsymbol{x})$ in (5) with $I_{\text {modified }}(\boldsymbol{x})$. When the original intensity image is compared to the modified intensity image in Fig. 9, a nonlinear filtering effect can be observed on the intensity image that further enhances the lumen boundary.

3) Stopping Criteria: The lumen segmentation usually converges after 5 to 50 iterations. During the segmentation of the lumen, the energy undergoes oscillations due to speckle in the luminal area; therefore, using the energy as the stopping criterion may cause the evolution to get stuck in local extrema. Instead, we observed that the contour starts oscillating and particularly in the rectangular grid, the contour starts to move up and down when it is close to convergence. This corresponds to an expanding and diminishing of the area that is enclosed by the segmented contour. Therefore, after ten oscillations of the area size, the algorithm is stopped.

A sample evolution of the shape weights, hence the segmentation, over the artificial evolution time $t$ is depicted in Fig. 10(c)-(f).

\section{B. Media-Adventitia Segmentation}

In this section, we describe the segmentation of the boundary between media and adventitia tissues. Statistical measurements around the media and adventitia border are not reliable because the intensity above and below the $\mathrm{m}$-a contour do not display particular regional patterns. We, therefore, need to analyze the local characteristics around the m-a interface. In IVUS images, the media is observed as a thin black line, whereas the adventitia tissue has high echogenicity characteristics and appears very bright. We take advantage of this anatomical observation by employing edge information. We use gradient information for the initialization as well as for the evolution of the shape. However, a pointwise gradient is not very useful because of the 


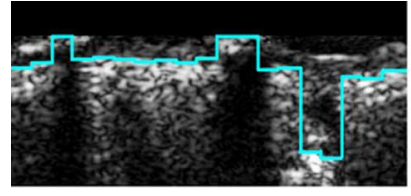

(a)

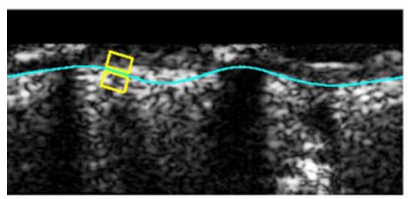

(c)

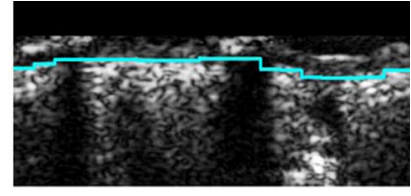

(b)

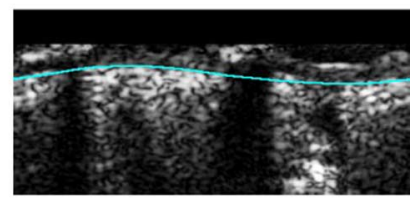

(d)

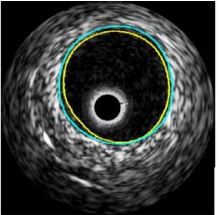

(a)

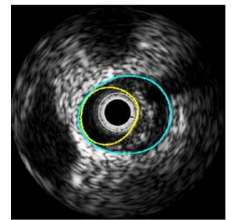

(d)

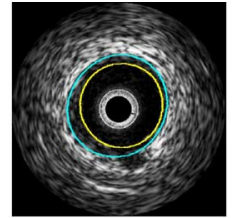

(b)

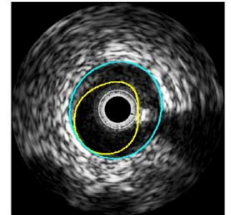

(e)

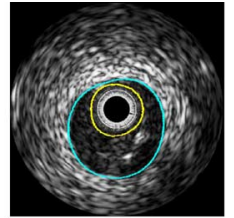

(c)

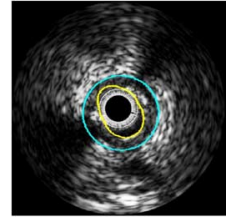

(f)
Fig. 11. Segmentation of m-a. (a) Max intensities contour. (b) Median filtered contour. (c) Initial shape pose with gradient window. (d) Evolved contour (after six iterations)

inherent speckle noise present in IVUS images. To overcome noise effects, we define a smoother version of the gradient as the difference between the average intensity of a box region above and below the current pixel position

$$
\begin{aligned}
G_{\text {smooth }}(x, y)= & \frac{1}{(2 d x+1) d y}\left(\sum_{i=-d x}^{d x} \sum_{j=1}^{d y} I(x+i, y+j)\right. \\
& \left.-\sum_{i=-d x}^{d x} \sum_{j=-d y}^{-1} I(x+i, y+j)\right)
\end{aligned}
$$

with $2 d x+1$ being the width and $d y$ the height of the gradient window.

1) Initialization of the Shape Pose: Since we use gradientbased energy for the shape evolution, a good initialization of the first shape pose, which is not very far away from the final $\mathrm{m}$-a contour, is required. We divide the rectangular image into $n$ columns $\left(n=25,=14.4^{\circ}\right.$ angular intervals set through experiments), and find the position of maximal smooth gradient for every column. This will give us a first initialization of the $\mathrm{m}$-a contour [see Fig. 11(a)]. A median filter that is passed over this initial contour eliminates eventual noise due to small artifacts or openings as shown in Fig. 11(b). We then project the median filtered contour onto our shape space to obtain our initial shape pose, which is a smooth contour [see Fig. 11(c)].

2) Evolution of the Shape: For the evolution of the m-a contour, we utilize a modified form of the edge-based energy [22]

$$
E^{a}\left(\boldsymbol{w}^{a}\right)=\oint_{\boldsymbol{C}} G(s) d s
$$

where $s$ is a parameter along the contour $\boldsymbol{C}$. We compute the gradient as the difference between the average intensity of two oriented windows above and below the contour, as shown in Fig. 11(c). We then derive the ODE for the m-a contour, discarding the unnecessary curvature terms

$$
\frac{\partial w_{i}^{a}}{\partial t}=\int_{\boldsymbol{C}} \nabla G(\boldsymbol{x}) \boldsymbol{U}_{i}^{a} d \boldsymbol{x}
$$

Fig. 12. Some examples of automatically computed lumen and m-a contours, including the following cases. (a) Healthy artery without plaque. (b) Centered catheter. (c) Small lumen with spurious noise between lumen and m-a. (d)-(f) Calcification with black shadow.

with $\nabla G$ representing the smoothed oriented edge gradient

$$
\nabla G(\boldsymbol{x})=G_{\text {oriented }}(\boldsymbol{x}-\beta \boldsymbol{n})-G_{\text {oriented }}(\boldsymbol{x}+\beta \boldsymbol{n})
$$

where $\beta$ is the scope of the gradient, and $\boldsymbol{n}$ is the normal vector to the contour at position $\boldsymbol{x}$. Since the gradient is taken along the contour, the inclination of the gradient is known to us. We, therefore, take the gradient as the difference between the average intensity of two oriented windows above and below the contour, as shown in Fig. 11(c)

$$
\begin{aligned}
G_{\text {oriented }}(\boldsymbol{x})= & \frac{1}{(2 d x+1) d y}\left(\sum_{i=-d x}^{d x} \sum_{j=-d y}^{-1} I(\boldsymbol{x}+i \boldsymbol{u}+j \boldsymbol{n})\right. \\
& \left.-\sum_{i=-d x}^{d x} \sum_{j=1}^{d y} I(\boldsymbol{x}+i \boldsymbol{u}+j \boldsymbol{n})\right)
\end{aligned}
$$

where $\boldsymbol{u}$ is the unit tangent vector and $\boldsymbol{n}$ the unit normal vector to the contour at point $\boldsymbol{x}$.

In addition, we incorporate an anatomical constraint to the evolution of the $\mathrm{m}$-a so that the minimum distance to the lumen is $0.2 \mathrm{~mm}$.

3) Stopping Criteria: The same stopping criteria as for the lumen segmentation is also used for the $\mathrm{m}$-a segmentation. The evolution is stopped after the area size of the $\mathrm{m}$-a oscillates more than ten times.

Sample segmentation results are shown in Fig. 12 for both the lumen and m-a contours.

\section{FEATURE DETECTION}

The segmentation algorithm presented in the previous section can overcome minor calcifications and branch openings that are not very wide and side branches that do not connect to the lumen, due to the restricted shape space and by median filtering of the initial contour. When the features are too wide though, the segmentation results may be erroneous. In these situations, we need to detect the features and interpolate the contour. This section focuses on detecting calcifications and 

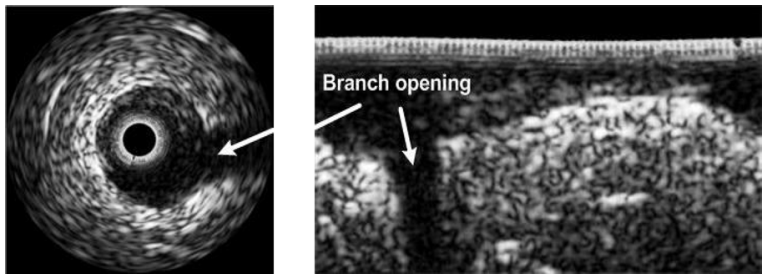

Fig. 13. Branch opening in display and rectangular domain.

branch openings, and incorporating that knowledge into the ma contour extraction.

\section{A. Side Branches}

Side branches are identified as the openings formed when the vessel being imaged bifurcates. This is visualized as an area of dark intensity extending from the lumen in the near field toward the far field. The intensity pattern is detectable in the rectangular image domain as a dark intensity segment extending in the vertical direction (see Fig. 13).

To detect side branches, the image is divided into $n$ columns, similar to the m-a initialization, and columns of dark intensity are sought. For every column, a square of the width of a column is shifted from top to bottom. The maximum smoothed intensity for every column is noted. From these intensities, the maximum smoothed intensity over the whole image is obtained. A column is classified as a branch opening, if its maximum smoothed intensity is smaller than $20 \%$ of the overall maximum intensity. At the positions of branch opening, the initial $\mathrm{m}$-a contour is interpolated linearly. Note that a linear interpolation in the rectangular domain corresponds to a circular interpolation in the display domain. Once the initial $\mathrm{m}$-a contour is interpolated correctly, it is median filtered and the initial shape pose is found. The evolution method of the $\mathrm{m}$-a shape is not affected by the side branch feature, because in the region of the dark branch opening, there is no high gradient by which the contour can be distracted. Fig. 14 shows the flowchart of our overall method depending on the side branch feature. In the cases of branch opening, the evolution of the luminal contour is delimited by the m-a contour, as shown in Fig. 15. Otherwise, the evolution of the m-a contour will be constrained by the lumen contour.

\section{B. Calcifications}

1) Anatomical Observations: Calcium deposits are places along the vessel wall where minerals have collected to form dense pockets. In IVUS, these deposits can be recognized by the presence of a bright echo along the lumen and shadowing of the far-field structures. In the rectangular domain, the bright calcification can be seen below the lumen and above the m-a contour. The shadow appears as a dark region below the calcification. In Fig. 16, a typical calcification with the shadowing is shown in the display and rectangular domain. The size of the shadow depends on the distance to the catheter. The closer the calcification is to the catheter, the bigger the shadow will appear. In the zone of the shadow, the $\mathrm{m}$-a border is missing. We also notice that the shadow is narrower than the calcification.

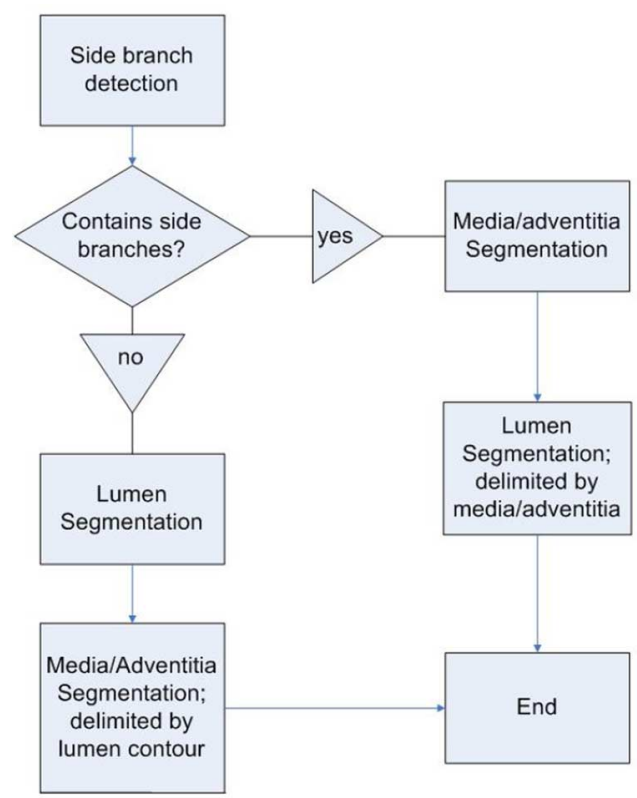

Fig. 14. Order in which the segmentation algorithms are executed depends on whether branch openings have been detected or not.
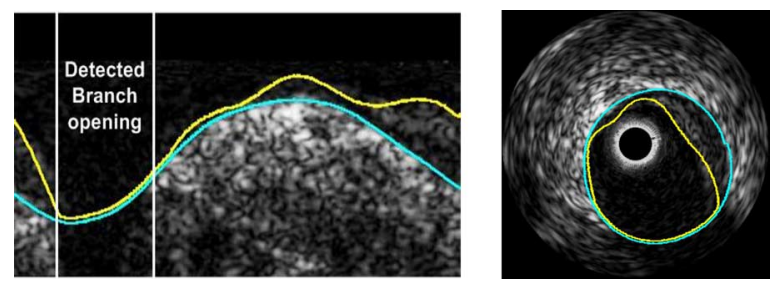

Fig. 15. Interpolated branch opening in the rectangular and display domain; lumen is delimited by the m-a.
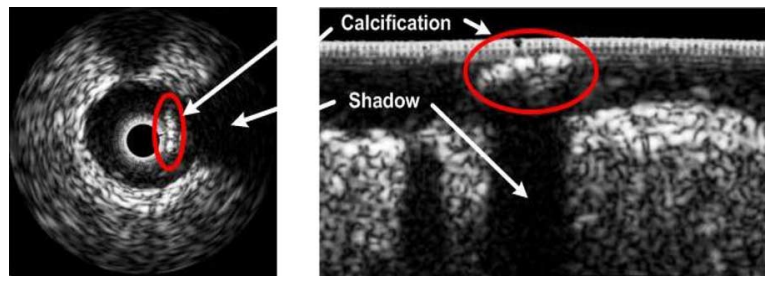

Fig. 16. Calcification with shadowing in display and rectangular domain.

2) Media-Adventitia Segmentation and Calcifications: During the initialization process of the $\mathrm{m}$-a contour, the rectangular image is divided into $n$ columns. The positions of maximal smoothed gradient are taken as an initial contour. At the calcified regions, the high gradient will be detected at the upper border of the calcification rather than at the m-a contour. Although median filtering worked for minor calcifications, it cannot treat the problems due to heavy calcifications. Fig. 17 shows how the detection and interpolation of calcifications is integrated in the segmentation of the m-a contour.

We explored detection of the bright calcified regions by locating the shadow that is caused by the calcification. This approach is the most frequently used one in literature, for instance as in [23] where adaptive thresholding is combined with shadow 


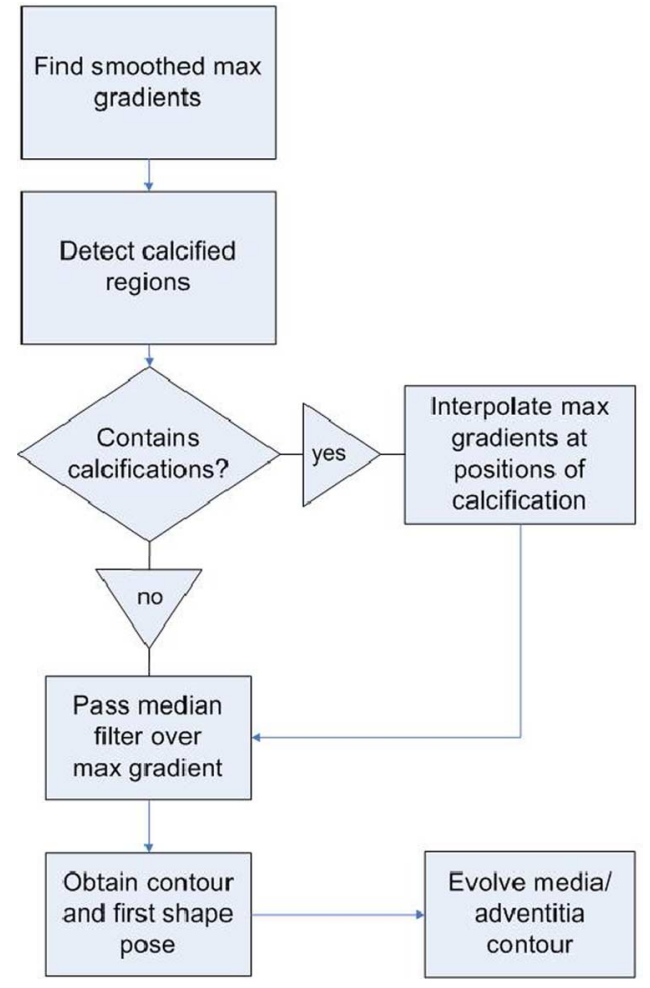

Fig. 17. Incorporation of the calcification detection into the m-a contour segmentation.

detection. A simple method to find shadows in the rectangular image is to look for dark intensities in the lower half of the image. Once the shadow is detected, we find where the $\mathrm{m}$-a contour needs to be interpolated. We encounter several problems: some calcifications do not leave a shadow or the shadow is too small to be detected. In some images, we observe a double layer: the tissue below the luminal border is brighter than the tissue below the $\mathrm{m}-\mathrm{a}$, but it is not big enough to leave a shadow. In addition, crucial parameters to be set are shadow intensity and size, which make the approach sensitive to parameter selection.

Another idea was to detect a jump of the maximal gradient contour at the beginning and at the end of the calcification; however, this method would be too much dependent on a parameter to determine how big the jump must be to indicate the beginning or end of a calcification. Therefore, further information in addition to the maximal gradient is required.

3) Proposed Approach To Detecting Calcifications: The m-a contour is visible at the start and end column of a calcification as can be observed in Fig. 18. Our goal is to detect those "hidden" gradients of the m-a contour. Fig. 19 depicts the intensity and smoothed gradient for the start column in Fig. 18 from top to bottom. We see that the global maximum gradient $C_{\max }^{a}$ is located at the upper edge of the calcification. At the lower edge of the calcification, there is a negative gradient minimum. The next positive gradient maximum below the calcification lies on the $\mathrm{m}$-a border. We can use this information to find the "hidden" $\mathrm{m}$-a gradients. The lower border of the calcification is found by detecting the gradient minimum $C_{\text {min }}^{a}$ below the global gradient maximum $C_{\max }^{a}$ and above the next positive gradient. The $\mathrm{m}$-a

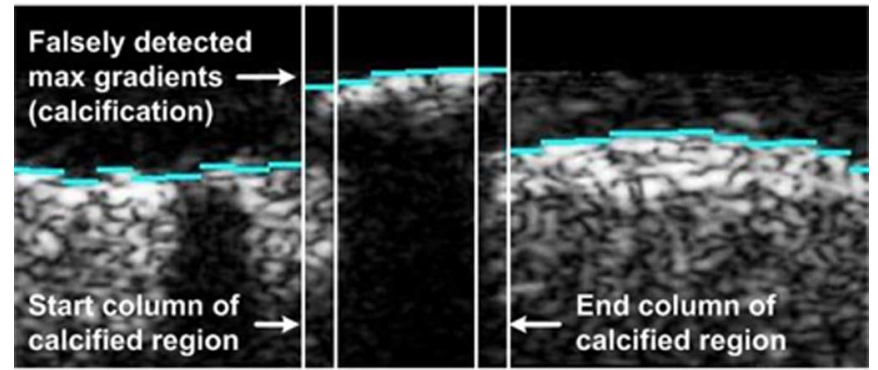

Fig. 18. Positions of global maximum gradients with the highlighted start and end columns.

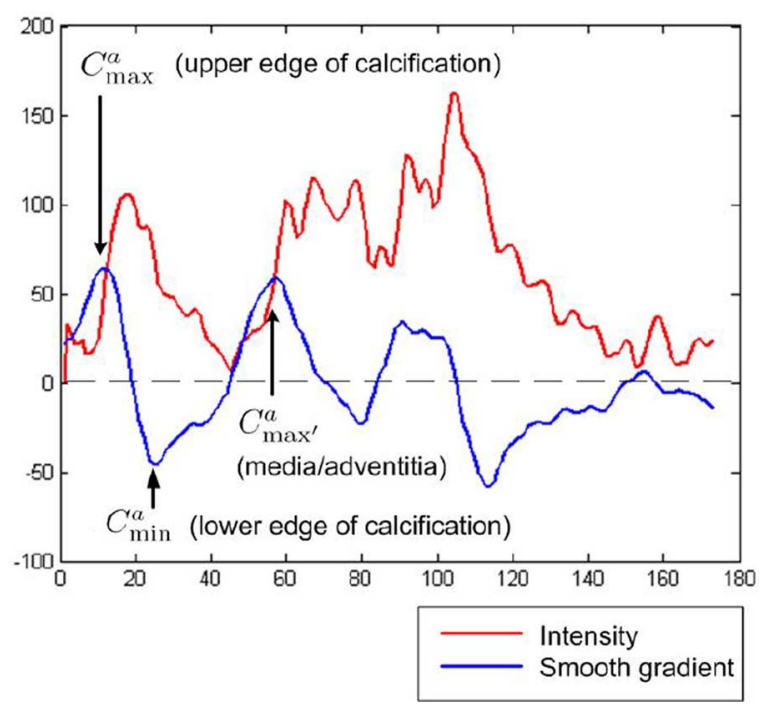

Fig. 19. Intensity and smoothed gradient from top to bottom of the start column in Fig. 18.

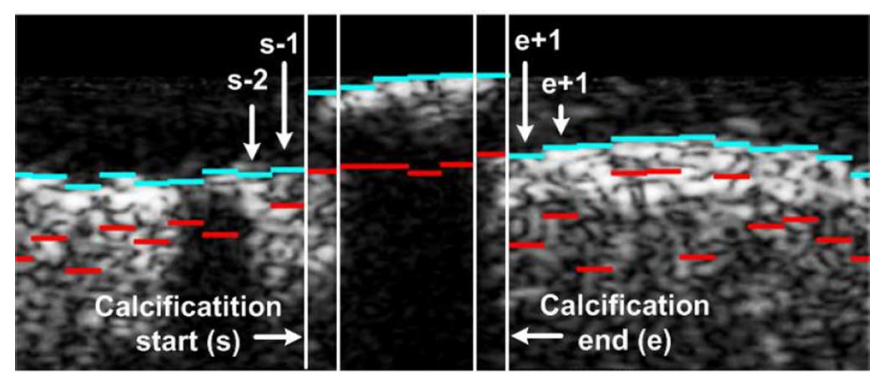

Fig. 20. Global max gradients $C_{\max }^{a}$ (in blue/light gray), second max gradients $C_{\text {max }^{\prime}}$ (in red/dark gray), and the start and end columns of the calcification.

border will be identified as the maximum gradient below the $C_{\mathrm{min}}^{a}$ and above the next negative gradient. We call this local gradient maximum $C_{\text {max }^{\prime}}^{a}$.

The positions of $C_{\max }^{a}$ and $C_{\max }^{a}$ for every column are shown in Fig. 20. We can see that $C_{\text {max' }}^{a}$ has been detected at the m-a contour that was "hidden" by the calcification. When the shadow of a calcification is big, the whole $\mathrm{m}$-a contour is not visible. Yet, at the start and end columns of the calcification, a local maximum gradient at the m-a contour can always be detected. After detecting all the columns that are a potential calcification start, we do the same for finding the potential calcification endings. 


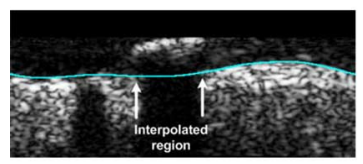

(a)

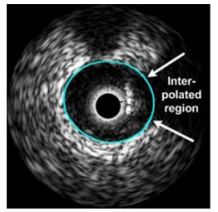

(b)

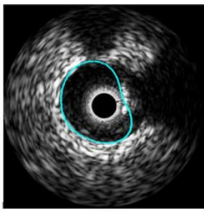

(c)
Fig. 21. (a) Interpolated and evolved $\mathrm{m}$-a contour in rectangular domain. Evolved m-a contour (b) with and (c) without detection and interpolation of the calcified region in the display domain.

The algorithm for detecting a potential end column is symmetric to the detection of the start column (see [24] for further details).

The maximum gradients contour from the start column $s$ to the end column $e$ will now be linearly interpolated between $C_{\max }^{a}(s-1)$ and $C_{\max }^{a}(e+1)$, as shown in Fig. 20. The obtained interpolated maximum gradient contour is then used to initialize the segmentation algorithm for the $\mathrm{m}$-a border. The evolved contour after seven iterations for the example in Fig. 20 can be seen in Fig. 21(a), and depicted in the display domain in Fig. 21(b). Without calcification detection, the unsuccessful result in Fig. 21(c) would be obtained.

\section{RESULTS AND DisCUSSIONS}

The lumen and m-a contours in our training and test datasets (explained in Section II-B) were segmented by an expert interventional cardiologist in our team, in order to provide the "ground truth" segmentation contours. We divided the 1919 test frames into two categories. In the first category of 1272 frames, with only small calcifications and branch openings, segmentation without the feature detection part is tested. The second test set containing all the test frames also includes large calcifications and branch openings, and is used to test the segmentation combined with the feature detection.

\section{A. Segmentation Without Feature Detection}

Fig. 12 demonstrated our results for several scenarios. We found that our $\mathrm{m}$-a algorithm works very well when there are no strong features such as a large calcification or a large side branch opening. With minor calcification and side branches, the segmentation is fairly successful due to the nicely constrained shape space in which our segmentation takes place. Because of this, even if there are openings, noise or other artifacts, the contour stays as a closed smooth contour, and can achieve meaningful results. Table I depicts several performance measures such as false positive rate $[\mathrm{FPR}=\mathrm{FP} /(\mathrm{FP}+\mathrm{TN})$ : false positive pixel counts normalized by total number of false instances, which are pixels that do not belong to the lumen or vessel], false negative rate $[\mathrm{FNR}=\mathrm{FN} /(\mathrm{FN}+\mathrm{TP})$ : false negative pixel counts normalized by the total number of positive instances, which belong to the lumen or vessel], overlap ratio $[=\mathrm{TP} /(\mathrm{TP}+\mathrm{FN}+\mathrm{FP})]$, mean and maximum distance and area difference between the manually segmented and automatically segmented contours over the test dataset. We present the average values with the confidence intervals (1.96 times the standard deviations assuming a normal distribution) with em- ploying an extreme outlier rejection, which eliminates those data values which are beyond the outer fences. It can be observed that our algorithm achieved a very low false positive rate of less than $1 \%$ (errs on the side of under segmentation), and relatively low false negative rates for the lumen $(3.01 \% \pm 5.35)$ and for the media $(5.35 \% \pm 7.86)$. The expert contour overlap ratio with our contours is $88.05 \% \pm 10.66$ for the lumen and $92.23 \% \pm 6.27$ for the $\mathrm{m}-\mathrm{a}$ borders. Average area difference between the expert and our contours is $0.60 \pm 0.99 \mathrm{~mm}^{2}$ for the lumen and $0.49 \pm 0.76 \mathrm{~mm}^{2}$ for the $\mathrm{m}$-a. Between the expert and our contours, the mean distance statistics are $0.08 \pm 0.10 \mathrm{~mm}$ for the lumen and $0.07 \pm 0.08 \mathrm{~mm}$ for the $\mathrm{m}-\mathrm{a}$, and the maximum distance statistics are $0.44 \pm 0.76 \mathrm{~mm}$ for the lumen and $0.84 \pm 1.74 \mathrm{~mm}$ for the $\mathrm{m}-\mathrm{a}$. Fig. 22 shows the Bland-Altman plots [25] that are scatter plots of the difference versus average between two measurements, here the expert contour areas and the autosegmented contour areas. The results are shown for both the lumen and the m-a contours and $95 \%$ of the difference is less than two standard deviations as shown, and the mean of the differences is close to 0 . These are indicators for the consistency of the method.

\section{B. Segmentation With Feature Detection}

We utilized our segmentation method with feature detection for the more challenging second test dataset. Fig. 23 demonstrates our segmentation results with feature detection for several frames. Wide branch openings and heavy calcifications are detected, taking advantage of anatomical characteristics. Incorporating the feature information into the $\mathrm{m}$-a contour extraction enhances our segmentation method. The segmentation of the lumen contour is improved as well, by constraining the lumen by the media contour in case of branch openings.

We obtain Table II from the calculations using our segmentation algorithm with the feature detection. Due to the much more challenging scenarios of this IVUS test dataset, without incorporating the feature detection part of the algorithm, we would expect a deterioration in the algorithm performance. However, with the incorporation of the more sophisticated feature detection algorithm, we obtained statistical error measures as good as for those of the less challenging test dataset. For instance, the false positive rates remained similar to before $(0.73 \pm 1.15$ for lumen and $0.52 \pm 1.08$ for $\mathrm{m}-\mathrm{a})$, and false negative rates slightly increased (4.09 \pm 7.78 for lumen and $5.55 \pm 9.20$ for $\mathrm{m}-\mathrm{a})$. The overlap ratio and mean distance measures were similar to before, however, area difference error increased slightly and maximum distance between the expert contours and our results increased $(0.65 \pm 1.27 \mathrm{~mm}$ for lumen and $0.98 \pm 1.78 \mathrm{~mm}$ for $\mathrm{m}$-a). This is possibly due to presence of mild outliers in the dataset and they would cause more extreme maximum distance values between the expert contours and our results in more difficult image conditions. Fig. 24 depicts histogram of radial distances between the expert and autosegmented contours.

\section{Implementation Details}

Most of the parameters used in our algorithm are not critical and their heuristically set values work for all the experiments. 
TABLE I

Performance Measures for the Test Dataset 1: False Positive Rate, False Negative Rate, Overlap Ratio, Mean and Maximum Distance,

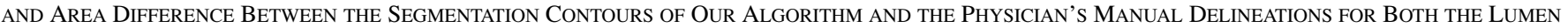
and the M-a Contours, Averaged Over the Test Dataset (Mean Value \pm 1.96 Standard Deviation Value Assuming a Normal Distribution)

\begin{tabular}{|c||c|c|c|c|c|c|}
\hline Mean \pm 1.96 Std & FPR $(\%)$ & FNR $(\%)$ & Overlap Ratio (\%) & Mean Distance $(\mathrm{mm})$ & Max Distance $(\mathrm{mm})$ & Area $\left(\mathrm{mm} \mathrm{m}^{2}\right)$ \\
\hline Lumen & $0.75 \pm 1.06$ & $3.01 \pm 5.35$ & $88.05 \pm 10.66$ & $0.08 \pm 0.10$ & $0.44 \pm 0.76$ & $0.60 \pm 0.99$ \\
\hline Media/Adv. & $0.46 \pm 0.90$ & $5.35 \pm 7.86$ & $92.23 \pm 6.27$ & $0.07 \pm 0.08$ & $0.84 \pm 1.74$ & $0.49 \pm 0.76$ \\
\hline
\end{tabular}

The pixel size is $25 \times 25 \mu^{2}$, average lumen cross-sectional area is $8.13 \mathrm{~mm}^{2}$, and average vessel cross-sectional area is $13.08 \mathrm{~mm}$.

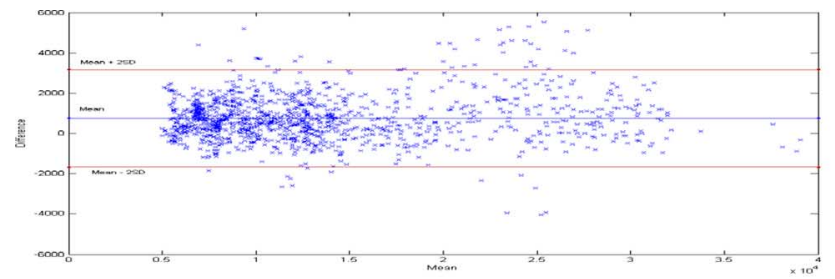

(a)

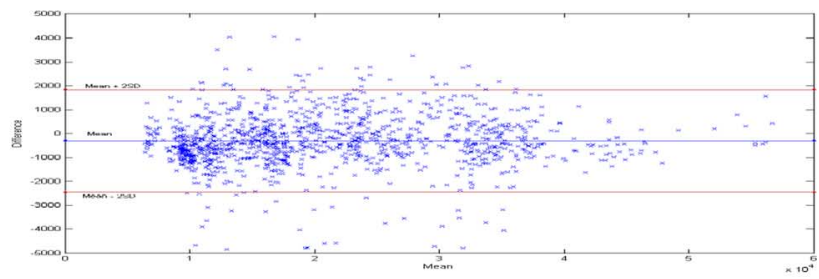

(b)

Fig. 22. Bland-Altman plots to indicate consistency between expert and auto-segmented contours.(a) Lumen area. (b) m-a Area.

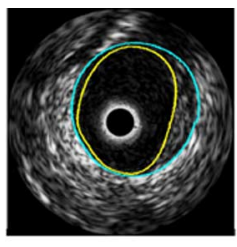

(a)

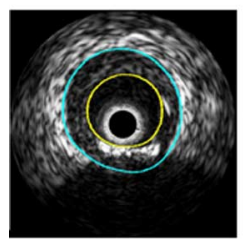

(d)

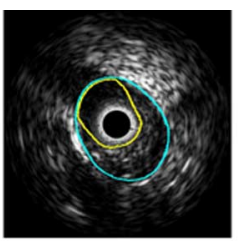

(b)

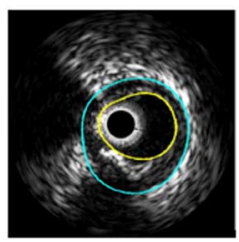

(e)

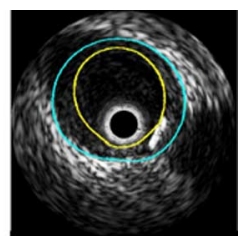

(c)

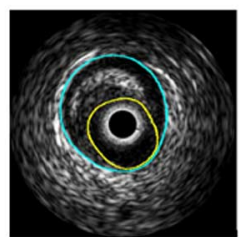

(f)
Fig. 23. Several examples of automatically computed lumen and $\mathrm{m}$-a contours with feature extraction, including the following cases. (a) and (b) Branch openings. (c) Branch opening and calcification. (d) Heavy calcification with black shadow. (e) Two calcifications. (f) Bright plaque region (calcification without black shadow).

Some details are given as follows: segmentation training utilized six eigen modes, momentum term weight $\delta=0.2$, time step $\alpha=600$, stopping criterion for the shape update equations is ten oscillations for the area size, Parzen window estimator kernel size $\sigma$ is chosen as 10 , scope of the smoothed gradients is 10 , median filter size is 7 . We note that we did not change these parameters for the experiments, even when working with the $40 \mathrm{MHZ}$ probe data. Normally, the pixel-based parameters should be scaled according to the resolution of the pullback. However, the advantage of our algorithm is that the computations are taking place in the rectangular domain resampled to a fixed number of pixels both in angular and radial directions. This leads to an inherent normalization operation on the images.

The algorithm speed performance is tested on an Intel Pentium $4,2.4 \mathrm{GHz}$ processor machine, where the training phase of the shape space and the intensity model is done off-line only once, and the obtained modes are then saved. The automatic segmentation of the lumen and $\mathrm{m}$-a contours takes between 5 and $10 \mathrm{~s}$ per image, and the feature detection time is less than $1 \mathrm{~s}$. We note that we have not done any code optimizations so far, and we expect a big potential speed up of the algorithm because it utilizes ODEs for the main update of the contour representation, and this part can be parallelized in hardware or graphics processing unit (GPU) based programming. This will be part of our future research for the physicians' interactive usage of our system.

\section{D. $40 \mathrm{MHZ}$ IVUS Probe}

With a $20 \mathrm{MHz}$ IVUS imaging probe, it can be observed that a fair amount of speckle noise is present in the lumen over the pullback. However, a higher frequency probe at $40 \mathrm{MHz}$ will produce more speckle noise. In this case, the lumen segmentation has to be retuned by computing a new intensity probability distribution. Using eight in vivo pullbacks of LAD, RCA, and LCX, acquired with a Boston Scientific Galaxy probe at 15 frames/s with $0.5 \mathrm{~mm} / \mathrm{s}$ using a $40 \mathrm{MHz}$ transducer, we retrained our probability distributions over a small set of 50 labeled frames. We again utilized the lumen contour segmentation equation (5) with the two new probability distributions $P_{\text {in }}$ and $P_{\text {out }}$.

In $40 \mathrm{MHz}$ probe pullbacks, the $\mathrm{m}$-a border again appears as the border between the thin dark media line and the bright adventitia tissue; therefore, the segmentation of the m-a contour did not require a modification. In addition, compared to the luminal border, the $\mathrm{m}$-a border has better contrast; therefore, we delimit the luminal contour by the $\mathrm{m}$-a border in this scenario.

We obtained some fair results for both the lumen and $\mathrm{m}-\mathrm{a}$ contours; however, further exploration and adaptation of the algorithm (e.g., some parameter tuning for $40 \mathrm{MHz}$ case) and a larger training set of labeled frames are required. These points 
TABLE II

Performance Measures for the Test Dataset 2: False Positive Rate, False Negative Rate, Overlap Ratio, Mean and Maximum Distance, and Area Difference Between the Segmentation Contours of Our Algorithm With Feature Detection and the Physician's Manual

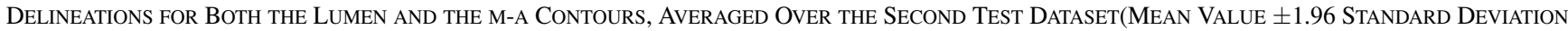
VAlue ASSUMing a Normal Distribution)

\begin{tabular}{|c||c|c|c|c|c|c|}
\hline Mean \pm 1.96 Std & FPR $(\%)$ & FNR $(\%)$ & Overlap Ratio $(\%)$ & Mean Distance $(\mathrm{mm})$ & Max Distance $(\mathrm{mm})$ & Area $\left(\mathrm{mm}^{2}\right)$ \\
\hline Lumen & $0.73 \pm 1.15$ & $4.09 \pm 7.78$ & $87.07 \pm 12.31$ & $0.09 \pm 0.12$ & $0.65 \pm 1.27$ & $0.64 \pm 1.15$ \\
\hline Media/Adv. & $0.52 \pm 1.08$ & $5.55 \pm 9.20$ & $90.57 \pm 10.66$ & $0.09 \pm 0.16$ & $0.98 \pm 1.78$ & $0.75 \pm 1.60$ \\
\hline
\end{tabular}

The pixel size is $25 \times 25 \mu \mathrm{m}^{2}$, average lumen cross-sectional area is $8.13 \mathrm{~mm}^{2}$, and average vessel cross-sectional area is $13.08 \mathrm{~mm}^{2}$.
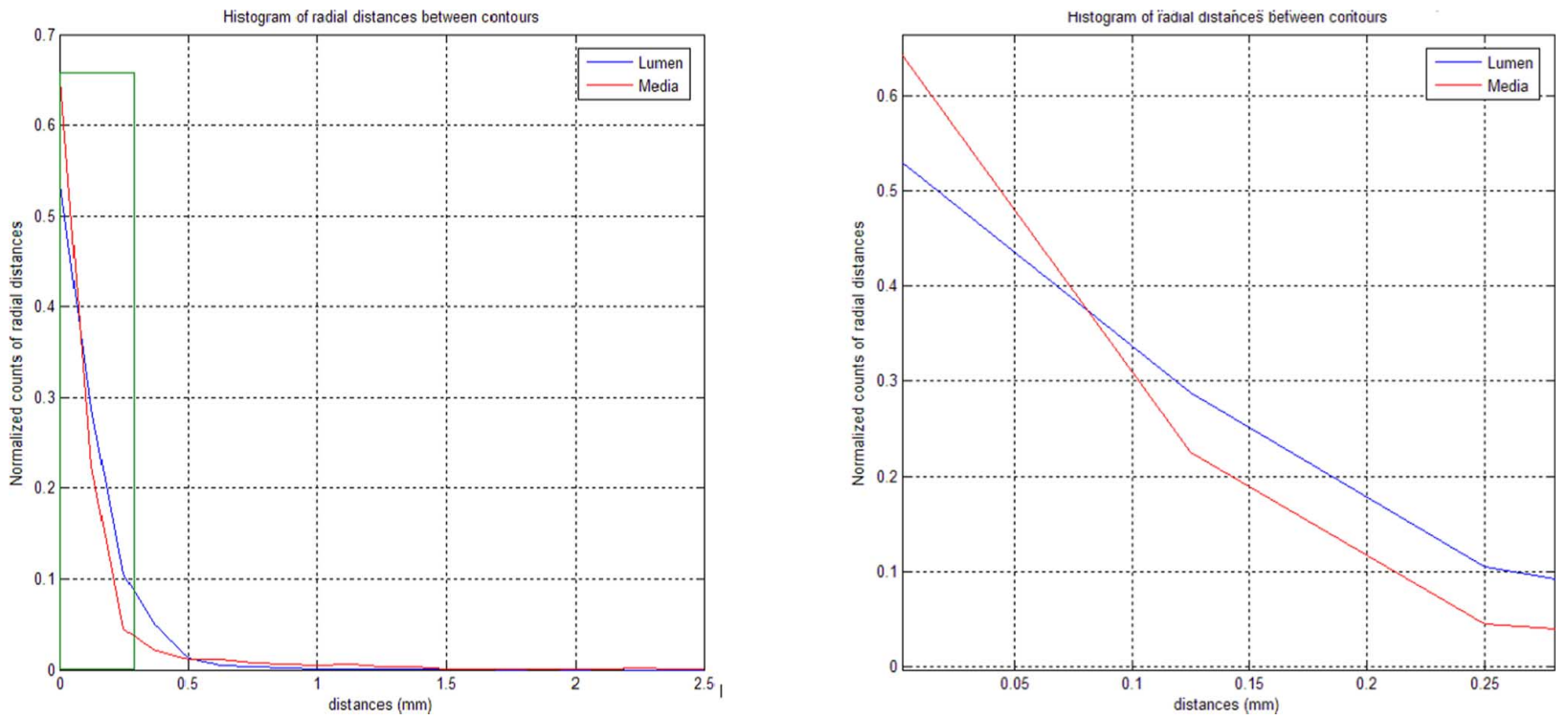

Fig. 24. (a) Normalized histogram of radial distances between the hand- and autosegmented contours both for lumen and m-a borders. (b) Histograms in (a) zoomed to visualize small distances.
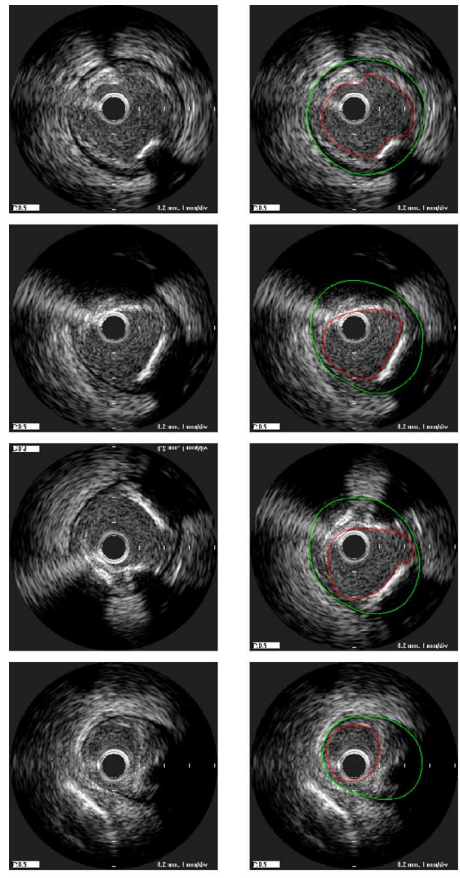

(a)

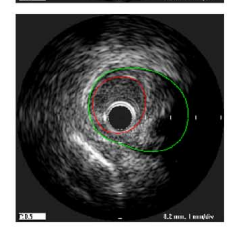

(b)
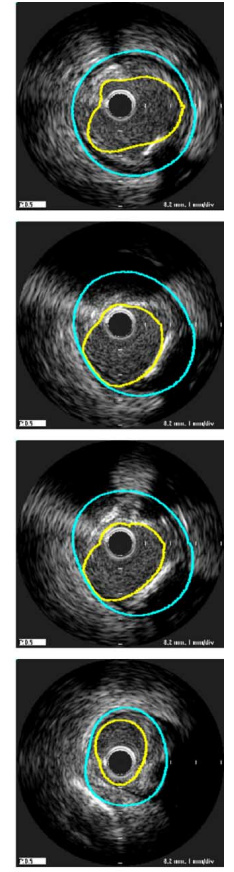

(c)

Fig. 25. Lumen and m-a segmentation on a pullback from the Boston Scientific Galaxy probe at $40 \mathrm{MHz}$. (a) Original image. (b) Image with doctor-drawn contours. (c) Segmentation result. High-frequency probe pullbacks pose more challenges to the algorithm, as can be seen in these images.

constitute our current ongoing studies. Some obtained results from one of the pullbacks can be seen in Fig. 25. The $40 \mathrm{MHz}$ pullbacks presented more challenging scenarios to the segmentation algorithm such as a more subtle difference in the patterns of inside the lumen versus inside the vessel, and less distinct $\mathrm{m}$-a borders. For instance, in Fig. 25 last row, the m-a segmentation is distracted by the bright pattern that caused high gradients outside the adventitia tissue farther away from the media border. Moreover, the contour in some cases could not conform to the real boundaries exactly. Therefore, further tuning and shape and intensity training will be required.

\section{CONCLUSION}

We presented a statistical shape model-based approach for segmenting arterial walls from IVUS images in the "resampled" rectangular domain. We constrained the lumen and m-a contours to a smooth, closed geometry, which increased the segmentation quality without any tradeoff with a regularizer term, yet with adequate flexibility. For segmenting the lumen contour, we utilized a nonparametric intensity model based on an image probability density energy, with global image measurements rather than pointwise measurements used in previous methods. The $\mathrm{m}-\mathrm{a}$ is segmented by employing edge information. We defined an oriented smooth gradient, which overcomes noise present in IVUS images. In addition, we developed a method to detect calcifications and branch openings, 
taking advantage of anatomical characteristics. Incorporating the feature information into the m-a contour extraction greatly enhances our segmentation method. We utilized data coming from IVUS probes at both 20 and $40 \mathrm{MHz}$, which displayed very different intensity characteristics, to expose the pros and cons of an IVUS segmentation technique and the challenges involved. Our results will be input to a postprocessing stage where one can calculate measurements such as lumen size, lumen radius, and wall radius, and will be further used in plaque analysis and 3-D reconstruction of the arterial wall. Due to unknown catheter position and motion, a geometrically correct 3-D reconstruction of the vessel is not possible given only an IVUS pullback; however, methods such as [26] that use the vessel geometry from biplane angiography in addition to IVUS pullbacks to overcome catheter motion can be utilized.

As part of our future work, we plan to focus on extending our algorithm to 3-D. We plan to take advantage of the continuity of images in the IVUS pullback sequences to enhance our algorithm. Extraction of stents and its incorporation into our segmentation algorithm also will be addressed.

\section{ACKNOWLEDGMENT}

The authors thank S. Biro and C. Castellanos for their help in data collection and import.

\section{REFERENCES}

[1] S. Nissen and P. Yock, "Intravascular ultrasound: Novel pathophysiological insights and current clinical applications," Circulation, vol. 103, pp. 604-616, 2001.

[2] W. Rosamond, K. Flegal, G. Friday, K. Furie, A. Go, K. Greenlund, N. Haase, M. Ho, V. Howard, B. Kissela, K. Kittner, D. Lloyd-Jones, M. McDermott, J. Meigs, C. Moy, G. Nichol, C. O’Donnell, V. Roger, J. Rumsfeld, P. Sorlie, J. Steinberger, T. Thom, S. Wasserthiel-Smoller, and Y. Hong, "Heart disease and stroke statistics-2007 update: A report from the American Heart Association Statistics Committee and Stroke Statistics Subcommittee," Circulation, vol. 115, no. 5, pp. 69-171, 2007.

[3] M. Sonka, X. Zhang, M. Siebes, M. Bissing, S. DeJong, S. Collins, and C. McKay, "Segmentation of intravascular ultrasound images: A knowledge-based approach," IEEE Trans. Med. Imag., vol. 14, no. 4, pp. 719-732, Dec. 1995.

[4] D. M. Herrington, T. Johnson, P. Santago, and W. E. Snyder, "Semiautomated boundary detection for intravascular ultrasound," in Proc. Comput. Cardiol., 1992, pp. 103-106.

[5] J. Dijkstra, G. Koning, J. Tuinenburg, P. Oemrawsingh, and J. Reiber, "Automatic border detection in intra vascular ultrasound images for quantitative measurements of the vessel, lumen and stent parameters," in Proc. Comput. Cardiol., 2001, pp. 25-28.

[6] G. Kovalski, R. Beyar, R. Shofti, and H. Azhari, "Three-dimensional automatic quantitative analysis of intravascular ultrasound images," $\mathrm{Ul}$ trasound Med. Biol., vol. 26, no. 4, pp. 527-537, 2000.

[7] J. Klingensmith, R. Shekhar, and D. Vince, "Evaluation of threedimensional segmentation algorithms for the identification of luminal and medial-adventitial borders in intravascular ultrasound images," IEEE Trans. Med. Imag., vol. 19, no. 10, pp. 996-1011, Oct. 2000.

[8] M. Plissiti, D. Fotidadis, L. Michalis, and G. Bozios, "An automated method for lumen and media-adventitia border detection in a sequence of IVUS frames," IEEE Trans. Inf. Technol. Biomed., vol. 19, no. 10, pp. 996-1011, Oct. 2000.

[9] E. Brusseau, C. deKorte, F. Mastik, J. Schaar, and A. van der Steen, "Fully automatic luminal contour segmentation in intracoronary ultrasound imaging-a statistical approach," IEEE Trans. Med. Imag., vol. 23, no. 5, pp. 554-566, 2004.

[10] M.-H. Cardinal, J. Meunier, G. Soulez, R. Maurice, E. Therasse, and G. Cloutier, "Automatic 3D segmentation of intravascular ultrasound images using region and contour information," in Proc. MICCAI (Lecture Notes in Computer Science), 2005, vol. 3749, pp. 319-326.
[11] E. Bovenkamp, J. Dijkstra, J. Bosch, and J. Reiber, "Multi-agent segmentation of IVUS images," Pattern Recog., vol. 37, no. 4, pp. 637-663, 2004.

[12] H. Zhu, Y. Liang, and M. Friedman, "IVUS image segmentation based on contrast," in Proc. SPIE Med. Imag.: Image Process, 2002, pp. 1727-1733.

[13] A. Mojsilovic, M. Popovic, N. Amodaj, R. Babic, and M. Ostojic, "Automatic segmentation of intravascular ultrasound images: A texture-based approach," Ann. Biomed. Eng., vol. 25, no. 6, pp. 1059-1071, 1997.

[14] C. Haas, H. Ermert, S. Holt, P. Grewe, A. Machraoui, and J. Barmeyer, "Segmentation of 3D intravascular ultrasonic images based on a random field model," Ultrasound Med Biol., vol. 26, no. 2, pp. 297-306, 2000.

[15] D. Gil, P. Radeva, J. Saludes, and J. Mauri, "Automatic segmentation of artery wall in coronary IVUS images: A probabilistic approach," in Proc. Int. Conf. Pattern Recog., 2000, pp. 4352-4355.

[16] M. Leventon, W. Grimson, and O. Faugeras, "Statistical shape influence in geodesic in active contours," in Proc. IEEE Conf. Comput. Vis. Pattern Recog., 2000, pp. 316-323.

[17] M. Rousson and D. Cremers, "Efficient kernel density estimation of shape and intensity priors for level set segmentation," in Proc. MICCAI, 2005, pp. 757-764.

[18] D. C. T. F. Cootes, C. J. Taylor, and J. Graham, "Active shape modelsTheir training and application," Comput. Vis. Image Understanding, vol. 61, no. 1, pp. 38-59, Jan. 1995.

[19] A. Tsai, A. Yezzi, W. Wells, C. Tempany, D. Tucker, A. Fan, W. Grimson, and A. Willsky, "A shape-based approach to the segmentation of medical imagery using level sets," IEEE Trans. Med.Imag., vol. 22, no. 2, pp. 137154, Feb. 2003.

[20] G. Unal, S. Bucher, S. Carlier, G. Slabaugh, T. Fang, and K. Tanaka, "Shape-driven segmentation of the arterial wall in intravascular ultrasound image," in Proc. MICCAI: The 1st Int. Workshop Comput. Vis. Intravasc. Intracardiac Imag. (CVII), G. Unal, I. Kakadiaris, G. Slabaugh, and A. Tannenbaum, Eds. 2006, pp. 51-58.

[21] N. Paragios and R. Deriche, "Geodesic active regions and level set methods for supervised texture segmentation," Int. J. Comput. Vis., vol. 46, no. 3, pp. 223-247, 2002.

[22] S. Kichenassamy, A. Kumar, P. Olver, A. Tannenbaum, and A. Yezzi, "Gradient flows and geometric active contours," in Proc. Int. Conf. Comput. Vis., 1995, pp. 810-815.

[23] E. dos Santos Filho, M. Yoshizawa, A. Tanaka, and Y. Saijo, "Segmentation of calcification regions in intravascular ultrasound images by adaptive thresholding," in Proc. CBMS, 2006, pp. 446-454.

[24] G. Unal, S. Bucher, G. Slabaugh, and T. Fang, "Feature detection in intravascular ultrasound images for segmentation of arterial wall,", Siemens Corporate Research, Princeton, NJ, Tech. Rep., 2006.

[25] J. Bland and D. Altman, "Statistical methods for assessing agreement between two methods of clinical measurements," Lancet, vol. 1, pp. 307310, 1986.

[26] A. Wahle, G. Prause, S. de Jong, and M. Sonka, "Geometrically correct 3D reconstruction of intravascular ultrasound images by fusion with biplane angiography-methods and validation," IEEE Trans. Med. Imag., vol. 18, no. 8, pp. 686-699, Aug. 1999.

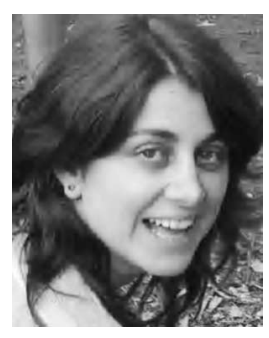

Gozde Unal (S'96-M'02-SM'08) received the Ph.D. degree in electrical engineering from North Carolina State University, Raleigh, in August 2002.

She was a Postdoctoral Fellow at the Georgia Institute of Technology, Atlanta. From Fall 2003 to 2007, she was a Research Scientist at Siemens Corporate Research, Princeton, NJ. She joined Sabanci University, Istanbul, Turkey, in Fall 2007, where she is currently an Assistant Professor. Her research interests include variational techniques with connection to information theory and probability theory, applications to various computer vision problems such as stereoscopic camera calibration, 2-D/3-D image segmentation and registration, filtering and enhancement, and stochastic particle systems. Her current research is focused on medical image analysis, segmentation, registration, and shape analysis techniques with applications to clinically relevant problems in magnetic resonance, computed tomography, Ultrasound, and intravascular images.

Dr. Unal was the General Chair of the 1st International Workshop on Computer Vision for Intravascular Imaging held in conjunction with the Medical Image Computing and Computer-Assisted Intervention (MICCAI) in 2006. 


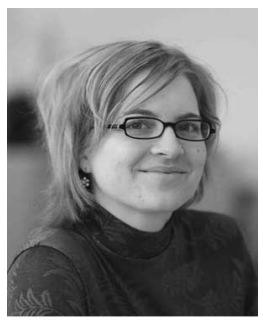

Susann Bucher received the M.Sc. degree in computer science from the Swiss Federal Institute of Technology, Lausanne, Switzerland, in 2006.

She wrote her Master Thesis at Siemens Corporate Research, Princeton, NJ about "Automatic Contour Segmentation in Intravascular Ultrasound Images". Since December 2006, she has been with the Avaloq Evolution AG, Zürich, Switzerland, as a Software Engineer, where he develops and maintains the Avaloq Banking System, an innovative and integrated IT platform which embraces modern banking practices.

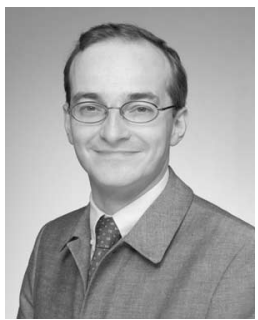

Stéphane Carlier received the M.D. degree and the degree in cardiology from the Free University of Brussels, Brussels, Belgium, in 1991 and 1996, respectively, and the Ph.D. degree in bioengineering from Erasmus University, Rotterdam, The Netherlands, in 2001.

$\mathrm{He}$ is currently the Director of medical affairs for Europe, Middle-East, and Africa for Cordis Corporation, a Johnson \& Johnson company, FL. $\mathrm{He}$ trained in interventional cardiology at the Thoraxcenter, Rotterdam. He was an Assistant Professor of Medicine at Columbia University, New York, where he was also the Director of the Intravascular Imaging and Physiology Corelab, Cardiovascular Research Foundation.

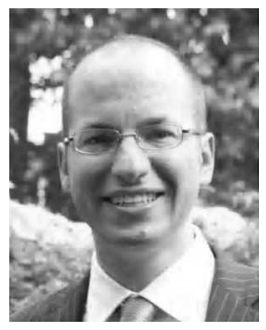

Greg Slabaugh received the B.Sc. degree in engineering physics from the University of Michigan, Ann Arbor, and the M.S. and Ph.D. degrees in electrical engineering from the Georgia Institute of Technology (the Center for Signal and Image Processing), Atlanta.

$\mathrm{He}$ is the Head of Research and Development at Medicsight, PLC-based in London, U.K. His current research interests include medical image processing, image and surface registration, segmentation, computer-aided detection, geometric modeling, adaptive filtering, PDEs, and level set methods.

Dr. Slabaugh is currently an Associate Editor of IEEE SIGNAL PROCESSING MAGAZINE. He co-organized the International Workshop on Computer Vision for Intravascular Imaging held in conjunction with Medical Image Computing and Computer-Assisted Intervention (MICCAI) 2006. The work in this paper was conducted while Greg was employed by Siemens Corporate Research.
Tong Fang received the M.Sc. degree in industrial engineering, the second M.Sc. degree in electrical and computer engineering, and the $\mathrm{Ph} . \mathrm{D}$. degree from Rutgers University, New Brunswick, NJ, in 1997, 1999, and 2000, respectively, and the third M.Sc. degree in management science from the University of Science and Technology, Hefei, China, in 1992.

He is currently a Research Scientist and the Manger of adaptive techniques R\&D program at Siemens Corporate Research, Princeton, NJ. His current research interests include medical image processing, geometric modeling, and visualization.

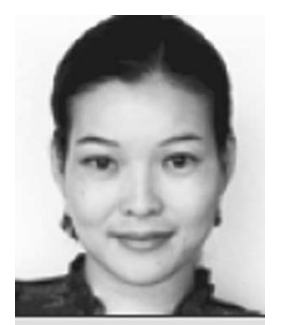

Kaoru Tanaka received the Graduate degree from Tokyo Womens Medical University, Tokyo, Japan, in 1996, and the M.D. and Ph.D. degrees.

She was an Imaging Fellow at the Cardiovascular Research Foundation and Columbia University Medical Center, New York, where he worked on intravascular ultrasound, cardiac magnetic resonance imaging, and cardiac computed tomography. From 1996 to 2001, she was a Clinical Fellow at Tokyo Womens Medical University, where she became an Assistant Professor of cardiology in 2001. 